\title{
Analysis of Three Compounds in Flos Farfarae by Capillary Electrophoresis with Large-Volume Sample Stacking
}

\author{
Hai-xia Yu, ${ }^{1}$ Zeng-Yan Hao, ${ }^{2}$ Lu Li, ${ }^{3}$ Ya-yun Huang, ${ }^{3}$ Hong-Fen Zhang, ${ }^{3}$ and An-jia Chen ${ }^{3}$ \\ ${ }^{1}$ Translational Medicine Research Center, Shanxi Medical University, Taiyuan 030001, China \\ ${ }^{2}$ Pharmacy Department, Shanxi Boai Hospital, Taiyuan 030001, China \\ ${ }^{3}$ School of Pharmacy, Shanxi Medical University, Taiyuan 030001, China
}

Correspondence should be addressed to Hong-Fen Zhang; zhanghf_2007@126.com and An-jia Chen; chenanjia888@163.com

Received 6 December 2016; Revised 10 April 2017; Accepted 12 June 2017; Published 5 September 2017

Academic Editor: Günther K. Bonn

Copyright (C) 2017 Hai-xia Yu et al. This is an open access article distributed under the Creative Commons Attribution License, which permits unrestricted use, distribution, and reproduction in any medium, provided the original work is properly cited.

\begin{abstract}
The aim of this study was to develop a method combining an online concentration and high-efficiency capillary electrophoresis separation to analyze and detect three compounds (rutin, hyperoside, and chlorogenic acid) in Flos Farfarae. In order to get good resolution and enrichment, several parameters such as the choice of running buffer, $\mathrm{pH}$ and concentration of the running buffer, organic modifier, temperature, and separation voltage were all investigated. The optimized conditions were obtained as follows: the buffer of $40 \mathrm{mM} \mathrm{NaH}_{2} \mathrm{PO}_{4}-40 \mathrm{mM}$ Borax-30\% v/v methanol ( $\mathrm{pH} 9.0$ ); the sample hydrodynamic injection of up to $4 \mathrm{~s}$ at $0.5 \mathrm{psi} ; 20 \mathrm{kV}$ applied voltage. The diode-array detector was used, and the detection wavelength was $364 \mathrm{~nm}$. Based on peak area, higher levels of selective and sensitive improvements in analysis were observed and about 14-, 26-, and 5-fold enrichment of rutin, hyperoside, and chlorogenic acid were achieved, respectively. This method was successfully applied to determine the three compounds in Flos Farfarae. The linear curve of peak response versus concentration was from 20 to $400 \mu \mathrm{g} / \mathrm{ml}, 16.5$ to $330 \mu \mathrm{g} / \mathrm{mL}$, and 25 to $500 \mu \mathrm{g} / \mathrm{mL}$, respectively. The regression coefficients were $0.9998,0.9999$, and 0.9991 , respectively.
\end{abstract}

\section{Introduction}

As is well known, capillary electrophoresis (CE) is powerful in separation and determination as a recently emerging liquid separation technology. Because of its high efficiency, high speed, ease of automation, ease of cleaning up contaminants, and analysis of sample and reagent in small volume, $\mathrm{CE}$ has been used to separate many different analytes, from small ions to macromolecules such as proteins and nucleic acids, and it is even used for the separation of particles and intact cells. In recent years CE usually is widely used in content determination and quality control of the traditional Chinese medicine herbs. However, the capillary tube has narrow internal diameter, which means that the $\mathrm{CE}$ technique generally has a low sample capacity and a short optical path length for online UV detection. All the above could decrease the detection sensitivity. Therefore, it is necessary to develop a suitable sample concentration step prior to separation to improve the limit of detection in CE.
There are two approaches to improve the detection sensitivity of CE, the off-column and online preconcentration method $[1,2]$. However, the off-line extraction introduces more steps before the $\mathrm{CE}$ procedure, increasing the risk of analyte losses. Most online concentration techniques are based on the velocity change in analyte between the sample zone and the separation zone and are easy to be operated. Therefore, online preconcentration, or stacking [3-6], is commonly used to improve the CE sensitivity, which increases the injected amount of analytes by using hydrodynamic or electrokinetic methods. Nowadays several methods of sample stacking have been used widely [7-10]. The methods include (i) normal stacking mode (NSM), (ii) field-amplified sample stacking (FASS), (iii) $\mathrm{pH}$-mediated stacking, (iv) acetonitrile stacking, and (v) large volume sample stacking (LVSS). Among all above, LVSS is a basic and widely adopted approach to enrichment [11-15], for it is simple and feasible and is suitable for detecting low levels of materials in drugs and the limit of detection can be achieved $\mathrm{ng} / \mathrm{mL}$. And 

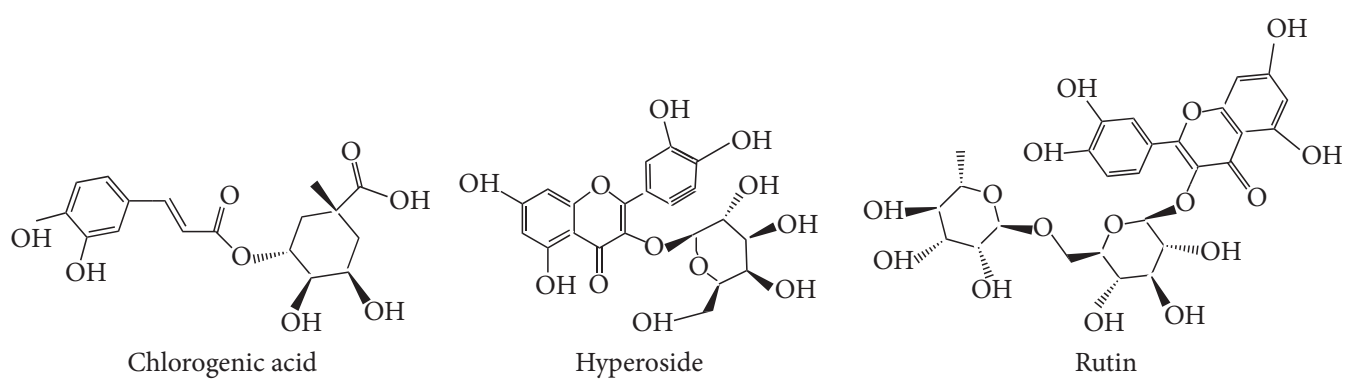

FIGURE 1: The structures of rutin, hyperoside, and chlorogenic acid.

the results of LVSS analysis are in high accuracy and good precision. It needs less sample consumption and has wide range of application. In this paper, an online concentration of LVSS in capillary electrophoresis is used to improve the detection sensitivity of the analysis.

Flos Farfarae is the flower buds of Tussilago farfara L., which is mostly produced in Shanxi, Henan, Gansu, and Hebei provinces and so on. As a widely used traditional Chinese medicine (TCM), Flos Farfarae has the therapeutic functions of relieving cough and reducing sputum. A variety of active compounds (triterpenoid saponins, sesquiterpenes, flavonoids, alkaloids, phthalic acid esters, chlorogenic acid, volatile oil, etc.) have been found in Flos Farfarae [16-19]. Rutin, hyperoside, and chlorogenic acid are the main active components in it and the structure of them is shown in Figure 1. In order to control the quality of Flos Farfarae better, establishing a method of the determination of rutin, hyperoside, and chlorogenic acid in Flos Farfarae is necessary. In many reports, high performance liquid chromatography (HPLC) is a good technique which has been used in the determination of compounds in Flos Farfarae [20-22]. Nowadays, Capillary electrophoresis gradually becomes a new technology in quality control of Flos Farfarae and other TCM [23-29].

In this study a new method combining the LVSS technique and high-efficiency CE was developed in order to analyze and determine three compounds in Flos Farfarae [30]. This method could relatively highly enhance the detection sensitivity of the compounds in Flos Farfarae and thus plays an important role in its quality control.

\section{Materials and Methods}

2.1. Reagents and Materials. Rutin, hyperoside, and chlorogenic acid were supplied by The National Institute For The Control of Pharmaceutical and Biological Products. Flos Farfarae were purchased from the Institutes for the drug Control of Taiyuan in Shanxi (S1), Linxian in Shanxi (S2), Anguo in Hebei (S3), Anyang in Anhui (S4), and Zhenzhou in Zhengzhou (S5). Borax and methanol were of analytical reagent grade. The stock solution containing rutin $5.0 \mathrm{mg} / \mathrm{mL}$ was prepared by dissolving $5.0 \mathrm{mg}$ rutin with $1 \mathrm{~mL}$ methanol and stored in a refrigerator $\left(4^{\circ} \mathrm{C}\right)$. The stock solution containing chlorogenic acid $4.0 \mathrm{mg} / \mathrm{mL}$ was prepared by dissolving $4.0 \mathrm{mg}$ chlorogenic acid with
$1 \mathrm{~mL}$ methanol. The stock solution containing hyperoside $3.3 \mathrm{mg} / \mathrm{mL}$ was prepared by dissolving $3.3 \mathrm{mg}$ hyperoside with $1 \mathrm{~mL}$ methanol. The desired concentration was prepared by diluting the stock solution with methanol prior to use. All solutions were filtered through a membrane $(0.45 \mu \mathrm{m})$ and then degassed (ultrasonic) for $2 \mathrm{~min}$ prior to use. The $\mathrm{pH}$ of buffers was adjusted with sodium hydroxide.

2.2. Apparatus and Operating Conditions for CE. The experiments were performed on a P/ACE MDQ system (AB Sciex, CA, USA) equipped with P/ACE diode-array detector. The system was controlled by P/ACE station software. The separation was carried out in a $75 \mu \mathrm{m} \times 50.2 \mathrm{~cm}$ fused-silica capillary (10.2 $\mathrm{cm}$ to the detector) (Yongnian Optical Fiber Factory, Hebei Province, China) with a cartridge of an $800 \times$ $100 \mu \mathrm{m}$ detector window.

The capillary was conditioned well by being flushed with $0.1 \mathrm{~mol} / \mathrm{L}$ sodium hydroxide, followed by water and methanol for $10 \mathrm{~min}$ at the pressure of $20 \mathrm{psi}$, respectively. Separation was carried out in reverse mode of electrode polarity, and applied voltage was maintained at $20 \mathrm{kV}$. The temperature of the capillary was maintained at $25^{\circ} \mathrm{C}$ and $364 \mathrm{~nm}$ was selected as the detection wavelength.

2.3. Sample Preparation. First, the sample was crushed into powder by using a Chinese herbal medicine crusher. The powder of samples (about $1.0 \mathrm{~g}$ ) was weighed and extracted with $30 \mathrm{~mL}$ methanol in an ultrasonic bath for $30 \mathrm{~min}$. Then the extracts were filtrated by filter paper and $0.45 \mu \mathrm{m}$ membrane filter, respectively, and analyzed under the optimized condition by CE.

2.4. Running Buffer for CE. The running buffer was prepared as follows: $5 \mathrm{~mL}, 80 \mathrm{mM} \mathrm{NaH}_{2} \mathrm{PO}_{4}$ was mixed with $5 \mathrm{~mL}$, $80 \mathrm{mM}$ borax in a $10 \mathrm{~mL}$ flask. And methanol of $3 \mathrm{~mL}$ was added to the above buffer. Thus the running buffer of $40 \mathrm{mM}$ $\mathrm{NaH}_{2} \mathrm{PO}_{4}-40$ mM Borax-30\% v/v methanol was obtained.

\subsection{Operating Conditions for Large-Volume Sample Stacking.} There are three main steps in the LVSS method [31-33]: first, the capillary was filled with the running buffer; then the standard or sample was injected by hydrodynamic injection at the pressure of 0.5 psi in short time (about 4 seconds); second, when the sample solution flows into the capillary after a certain length, reversing polarity voltage (about $25 \mathrm{kV}$ ) was 
applied; third, the sample was stacked at the interface between the buffer and the sample zone. When the current reached $95 \%$ of the original value, the applied voltage was switched to normal polarity, and the separation was finished.

\section{Results and Discussions}

3.1. Effect of Buffer Concentration and $p H$. In selecting the proper electrophoretic medium for CE, several characteristics of the solvents should be considered including viscosity, dielectric constant, electrical and thermal conductivity, selfdissociation constant, polarity, and boiling point. The commonly used running buffers included borate, phosphate, or acetate. Borate buffers usually gave better resolution of the analytes than the other buffers.

Through being investigated by phosphate, acetic acid, and borax buffer system, the mixture of borax and $\mathrm{NaH}_{2} \mathrm{PO}_{4}$ was discovered as the best buffer system, after peak shape and analysis time were considered comprehensively. Additionally, buffer concentration obviously affects the separation because it can influence the electroosmotic flow (EOF) and the viscosity of electrolyte. In order to obtain the best resolution, the concentration of buffer was investigated in the range from 20 to $50 \mathrm{mM}$. The resolution of the analytes increased with the buffer concentration increasing. This phenomenon may be attributed to the decreased EOF with increased ion strength (decreasing of zeta-potential). Considering the resolution and the running current, the buffer containing $40 \mathrm{mM}$ borax and $40 \mathrm{mMNaH}_{2} \mathrm{PO}_{4}$ was selected for the subsequent experiments.

The $\mathrm{pH}$ of buffer is also a governing factor in electrophoretic separation, because it determines the extent of ionization of each individual analyte and influences the properties of the inner surface of capillary. Separation systems show completely different selectivities when the $\mathrm{pH}$ values are different. The buffer solutions in this work were prepared by adjusting their $\mathrm{pH}$ value with sodium hydroxide. Considering the analysis time and resolution of the main peaks, $\mathrm{pH} 9.0$ was finally selected.

3.2. Effect of Organic Modifier. In some cases, organic modifier, such as methanol and acetonitrile, was found to effectively improve the separation selectivity, efficiency, and resolution. The use of organic modifier could improve resolution satisfactorily. In order to separate compounds, methanol should be introduced. The effect of the percentage of methanol on resolution was also investigated by adding methanol ranging from $15 \%$ to $40 \% \mathrm{v} / \mathrm{v}$ into the buffer. The maximum resolution between the three compounds was obtained when the percentage of methanol in the buffer was $30 \% \mathrm{v} / \mathrm{v}$.

In all, the $\mathrm{pH}$, nature, and concentration of buffer and nature and concentration of organic modifier were the main factors that influence the separation of CE. So the separation was attempted in the mixture of $40 \mathrm{mM}$ borax$40 \mathrm{mM} \mathrm{NaH}_{2} \mathrm{PO}_{4}$ containing $30 \% \mathrm{v} / \mathrm{v}$ methanol ( $\mathrm{pH} 9.0$ ) and we found that the results were satisfactory.
3.3. Effect of Applied Voltage. The applied voltage also can influence the migration times of analytes in CE. With increased voltage, the analysis time became shorter. In this paper, the effect of applied voltage was tested in the range of $10-30 \mathrm{kV}$. The results showed that the migration time of the compounds investigated was shortened with increased applied voltage. However, permanently physical damage of the capillary usually was caused by a high voltage in LVSS $[34,35]$; thus the following CE process was blocked. This is because, during the stacking process, Joule heating was generated mainly in the sample zone. As the sample zone was driven to move back to remove solvent by the EOF, the sample zone became narrower and narrower, which brought about Joule heating being focused on a small area close to the capillary inlet in the sample zone. Applying a high voltage would produce a high electric current; thus great Joule heating was generated in this narrow area and the capillary could be destroyed itself. In our experiment, when voltage reached $25 \mathrm{kV}$, the capillary was easy to be damaged. On account of the above, and through repeated experiments, $20 \mathrm{kV}$ was proper voltage to be applied for both sample stacking and next separation.

3.4. Effect of Injection Volume and Stacking Time. Analytes were injected into the capillary in different injection time ranging from 1 to $10 \mathrm{~s}$. During the injection step, when the injection time was longer than $4 \mathrm{~s}$, the sample zone would surpass the capillary window and even be pumped out of the outlet of the capillary. When the sample zone moved backwards in the stacking process, they were detected. This way, in the present LVSS method, the maximum available injection volume was the capillary volume between the inlet and the detection window [36, 37]. In our following experiment, an injection time of $4 \mathrm{~s}$ was used.

In order to improve the detection limits of the analytes, sample volume should be held as small as possible after stacking. And no extra dispersion should be produced. The optimum time of compounds stacking and sample matrix removal from the capillary should also be tested. In this study, the sample solution was made by hydrodynamic injection of up to $4 \mathrm{~s}$ at $0.5 \mathrm{psi}$; then $20 \mathrm{kV}$ voltage was used for stacking and the matrix removal time of $0.7 \mathrm{~min}$ (the current was $95 \%$ of its maximum value) was selected.

3.5. Linearity, Reproducibility, and LOD. Under the optimized conditions, a series of the standard solutions were tested to determine the linearity in this method. The linear regression equations and correlation coefficients are shown in Table 1 . The reproducibility of the analytes in the experiment was determined under the optimum conditions by repeated injecting standard solutions $(80 \mu \mathrm{g} / \mathrm{mL}, n=6)$. The relative standard deviations (RSD) of rutin, hyperoside, and chlorogenic acid were $2.5 \%, 2.9 \%$, and $2.0 \%$, respectively. The result indicated that precision of the instrument was good. The sample solution was injected discontinuously six times within $24 \mathrm{~h}$. The RSD values of peak area were $1.1 \%, 2.1 \%$, and $1.7 \%$, which indicated that the sample was stable in $24 \mathrm{~h}$ at room temperature. The limit of detection of rutin, hyperoside, 


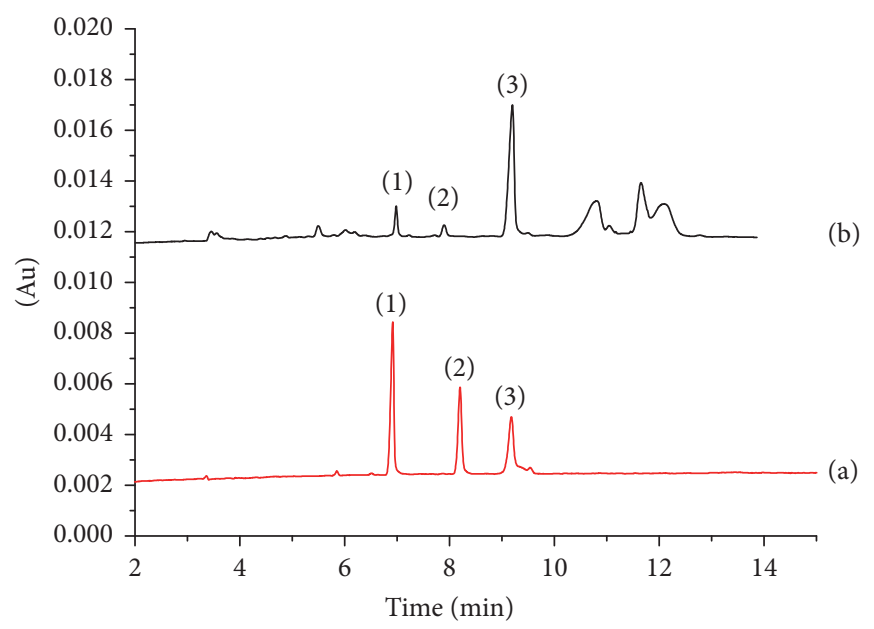

FIGURE 2: Electropherograms of (a) standard solution and (b) sample solution by CZE: (1) rutin; (2) hyperoside; (3) chlorogenic acid. CZE conditions: $40 \mathrm{mM} \mathrm{NaH}_{2} \mathrm{PO}_{4}-40 \mathrm{mM}$ borax ( $\mathrm{pH}$ 9.0)-30\% v/v methanol; voltage: $20 \mathrm{kV}$; injection volume: $0.5 \mathrm{psi}$, $4 \mathrm{~s}$; detection wavelength: $364 \mathrm{~nm}$; the concentration of individual compounds in standard sample: $200 \mathrm{ug} / \mathrm{mL}$.

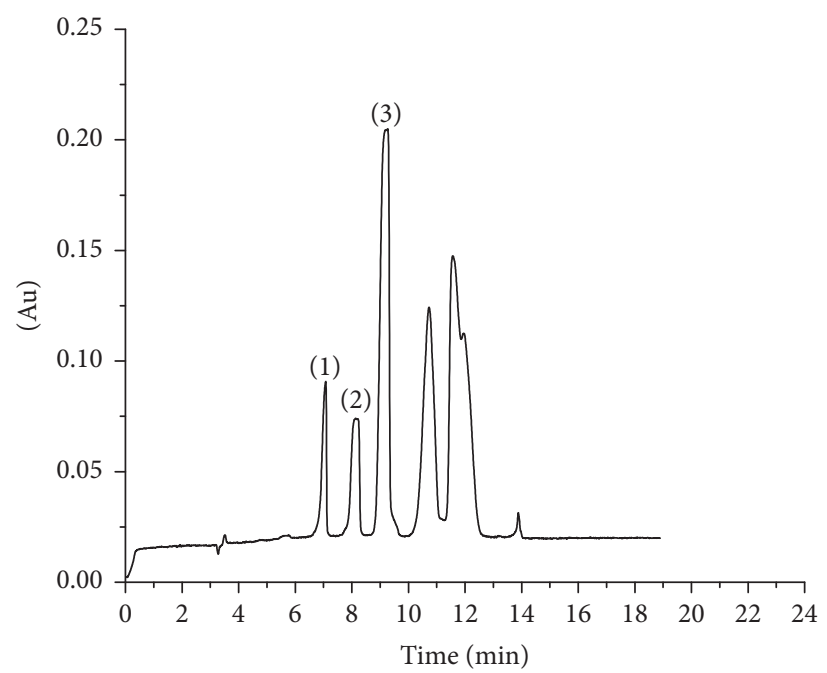

FIGURE 3: Electropherograms of sample solution by LVSS method: (1) rutin; (2) hyperoside; (3) chlorogenic acid. CE conditions: 40 mM $\mathrm{NaH}_{2} \mathrm{PO}_{4}-40 \mathrm{mM}$ Borax-30\% v/v methanol ( $\mathrm{pH} 9.0$ ); operating voltage: $20 \mathrm{kV}(+)-(-)$, the sample hydrodynamic injection of $4 \mathrm{~s}$ at $0.5 \mathrm{psi}$, water injection time: $40 \mathrm{~s}$; detection wavelength: $364 \mathrm{~nm}$.

and chlorogenic acid is $4 \mu \mathrm{g} / \mathrm{mL}, 5 \mu \mathrm{g} / \mathrm{mL}$, and $3 \mu \mathrm{g} / \mathrm{mL}$, respectively, before enrichment and $0.91 \mu \mathrm{g} / \mathrm{mL}, 0.94 \mu \mathrm{g} / \mathrm{mL}$, and $0.48 \mu \mathrm{g} / \mathrm{mL}$, respectively, after enrichment.

3.6. Determination and Accuracy of Three Compounds in Flos Farfarae. According to the procedures described in the experimental section, a method of the LVSS combining CE has been developed for the analysis of the three compounds in Flos Farfarae samples obtained from different regions. Electropherograms of standard solution and sample solution by CZE are shown in Figure 2. Electropherogram of sample solution by LVSS method is shown in Figure 3. There was 14-, 26-, and 5-fold enrichment of rutin, hyperoside, and chlorogenic acid according to ratio of peak areas of two methods. Peak identification was performed by a standard-addition method. Standard curve method was used to calculate the contents of samples. The assay results of five samples are summarized in Table 2. The contents of three compounds in Flos Farfarae are well in agreement with several previous reports $[38,39]$. Accurate amounts of standards were added to the powder drug of known content and then prepared to obtain sample solutions. Subsequently, the spiked sample solution was analyzed under the optimum conditions. The results are given in Table 3 . The results showed that the proposed method is reliable, accurate, and reproducible for the determination of three compounds in Flos Farfarae.

\section{Conclusion}

In conclusion, this study demonstrates that the use of CE combining a simple large-volume sample stacking method is effective in detecting three compounds in Flos Farfarae. 
TABLE 1: Calibration range, regression equation, and correlation coefficients.

\begin{tabular}{lccc}
\hline Analytes & Calibration range $(\mu \mathrm{g} / \mathrm{mL})$ & Regression equation & Regression coefficients \\
\hline Rutin & $20.0-400.0$ & $Y=408814 X+1475940$ & 0.9999 \\
Chlorogenic acid & $25.0-500.0$ & $Y=161158 X+254880$ & 0.9991 \\
Hyperoside & $16.5-330.0$ & $Y=207829 X-80269$ & 0.9999 \\
\hline
\end{tabular}

TABLE 2: Determination results of three compounds in Flos Farfarae $(n=3)$.

\begin{tabular}{lccc}
\hline $\begin{array}{l}\text { Source of } \\
\text { sample }\end{array}$ & $\begin{array}{c}\text { Content of rutin } \\
(\mathrm{mg} / \mathrm{g})\end{array}$ & $\begin{array}{c}\text { Content of } \\
\text { chlorogenic acid } \\
(\mathrm{mg} / \mathrm{g})\end{array}$ & $\begin{array}{c}\text { Content of } \\
\text { hyperoside }(\mathrm{mg} / \mathrm{g})\end{array}$ \\
\hline S1 & 5.1 & 9.2 & 2.3 \\
S2 & 2.4 & 1.9 & 2.1 \\
S3 & 3.1 & 11.1 & 3.3 \\
S4 & 5.8 & 10.0 & 3.4 \\
S5 & 4.7 & 2.4 \\
\hline
\end{tabular}

TABLE 3: Recovery of rutin, chlorogenic acid, and hyperoside in Flos Farfarae.

\begin{tabular}{|c|c|c|c|c|c|}
\hline Samples & Original amount (mg) & Added amount (mg) & Found amount (mg) & Average recovery (\%) & RSD (\%) \\
\hline Rutin & 1.273 & 1.300 & 2.578 & 100.3 & 0.1 \\
\hline Chlorogenic acid & 2.114 & 2.100 & 4.230 & 100.0 & 3.5 \\
\hline Hyperoside & 2.323 & 2.300 & 4.636 & 100.5 & 1.9 \\
\hline
\end{tabular}

Successful separation and accurate results were obtained. The compound was determined with high efficiency in a short period of time (less than ten minutes). The proposed method was validated and showed a good performance with respect to selectivity, precision, linearity, and accuracy. In all, compared with the previous methods by HPLC, HPCE showed advantages as follows: analysis time shortened greatly, sensitivity and limit of detection improved for simple and practical online enrichment, pretreatment is relatively simple, and there is less sample consumption.

Therefore, this new method is promising for the quality control of Flos Farfarae in the future.

\section{Conflicts of Interest}

The authors have declared no conflicts of interest.

\section{Authors' Contributions}

Hai-xia Yu and Zeng-Yan Hao contributed equally to the work.

\section{Acknowledgments}

This work was financially supported by National Natural Science Foundation of China (81241137) and Shanxi Key Subject Construction (029814).

\section{References}

[1] Z. Lin, X. Sun, Y. Lin, and G. Chen, "Highly sensitive analysis of four hemeproteins by dynamically-coated capillary electrophoresis with chemiluminescence detector using an offcolumn coaxial flow interface.", The Analyst, vol. 138, no. 8, pp. 2269-2278, 2013.
[2] G. L. Devault and M. J. Sepaniak, "Spatially focused deposition of capillary electrophoresis effluent onto surface-enhanced Raman-active substrates for off-column spectroscopy," Electrophoresis, vol. 22, no. 11, pp. 2303-2311, 2001.

[3] S. D. Arnett and C. E. Lunte, "Enhanced pH-mediated stacking of anions for CE incorporating a dynamic $\mathrm{pH}$ junction," Electrophoresis, vol. 28, no. 20, pp. 3786-3793, 2007.

[4] J. P. Quirino and S. Terabe, "Sample stacking of cationic and anionic analytes in capillary electrophoresis," Journal of Chromatography A, vol. 902, no. 1, pp. 119-135, 2000.

[5] J. P. Quirino, J.-B. Kim, and S. Terabe, "Sweeping: Concentration mechanism and applications to high-sensitivity analysis in capillary electrophoresis," Journal of Chromatography A, vol. 965, no. 1-2, pp. 357-373, 2002.

[6] J.-B. Kim, Y. Okamoto, and S. Terabe, "On-line sample preconcentration of cationic analytes by dynamic $\mathrm{pH}$ junction in capillary electrophoresis," Journal of Chromatography A, vol. 1018, no. 2, pp. 251-256, 2003.

[7] J. Horáková, J. Petr, V. Maier et al., “Combination of large volume sample stacking and dynamic $\mathrm{pH}$ junction for on-line preconcentration of weak electrolytes by capillary electrophoresis in comparison with isotachophoretic techniques," Journal of Chromatography A, vol. 1155, no. 2, pp. 193-198, 2007.

[8] Y. Y. Du, H. Q. Liu, L. Jia, and D. Xing, “Technology and application of capillary elec- trophoresis in the online sample enrichment," Journal of Instrumental Analysis, vol. 27, no. 1, pp. 103-112, 2008.

[9] L. F. Huang, M. He, B. B. Chen, and B. Hu, "Enrichment in capillary electrophoresis analy- sis technology and its application," Chinese Journal of Chromatography, vol. 32, no. 10, pp. 10661078, 2014.

[10] Y. X. Zhao, D. Q. Wang, X. M. Li, Q. Q. Yao, and L. V. Yuanqi, "Capillary electrophoresis on-line enrichment technology application in the analysis of flavonoids," Chemical Reagents, vol. 38, no. 4, pp. 323-326, 2016. 
[11] P. Tưma, "The use of polarity switching for the sensitive determination of nitrate in human cerebrospinal fluid by capillary electrophoresis with contactless conductivity detection," Journal of Chromatography A, vol. 1447, pp. 148-154, 2016.

[12] S. Almeda, L. Arce, and M. Valcárcel, "Combination of the solid-phase extraction and large-volume stacking with polarity switching in micellar electrokinetic capillary chromatography for the determination of traces of nonsteriodal antiinflammatory drugs in saliva," Electrophoresis, vol. 29, no. 14, pp. 3074-3080, 2008.

[13] D. Moreno-González, I. Lupión-Enríquez, and A. M. GarcíaCampaña, "Trace determination of tetracyclines in water samples by capillary zone electrophoresis combining off-line and on-line sample preconcentration," Electrophoresis, vol. 37, no. 9, pp. 1212-1219, 2016.

[14] Z. Zhu, X. Zhou, N. Yan, L. Zhou, and X. Chen, "On-line combination of single-drop liquid-liquid-liquid microextraction with capillary electrophoresis for sample cleanup and preconcentration: A simple and efficient approach to determining trace analyte in real matrices," Journal of Chromatography A, vol. 1217, no. 11, pp. 1856-1861, 2010.

[15] C. Quesada-Molina, A. M. García-Campaña, L. del OlmoIruela, and M. del Olmo, "Large volume sample stacking in capillary zone electrophoresis for the monitoring of the degradation products of metribuzin in environmental samples," Journal of Chromatography A, vol. 1164, no. 1-2, pp. 320-328, 2007.

[16] H. Xu, Y. Zhu, X. D. Yu, and H. Y. Chen, "Capillary electrophoresis in the online enrichment of the sample," Progress in Chemistry, vol. 17, no. 3, pp. 377-382, 2005.

[17] U. M. Seo, B. T. Zhao, W. I. Kim et al., "Quality evaluation and pattern recognition analyses of bioactive marker compounds from farfarae flos using HPLC/PDA," Chemical \& Pharmaceutical Bulletin, vol. 63, no. 7, pp. 546-553, 2015.

[18] Y. F. Liu, X. W. Yang, and B. Wu, "Studies on chemical constituents in the buds of Tussilago farfara," China Journal of Chinese Materia Medica, vol. 32, no. 22, pp. 2378-2381, 2007.

[19] Z. Z. Zhang, H. J. Zhi, X. M. Qin, and Z. Y. Li, "Chemical comparison of different Farfarae Flos by NMR-based metabolomic approaches," Acta Pharmacologica Sinica, vol. 50, no. 5, pp. 599604,2015

[20] W. Y. Zhang and T. S. Yang, "HPLC determination of chlorogenic acid and rutin contents in Flos Farfarae," Chinese Journal of Pharmaceutical Analysis, vol. 28, no. 12, pp. 2106-2108, 2008.

[21] Z. J. Ma and H. H. Dong, "Quantitative analysis and HPLC fingerprint of quercetin and kaempferide," Chinese Traditional and Herbal Drugs, vol. 40, no. 8, pp. 1305-1308, 2009.

[22] Y. F. Liu and X. W. Yang, "RP-HPLC determination of tussilagone in Flos Farfarae," Chinese Journal of Pharmaceutical Analysis, vol. 29, no. 1, pp. 31-34, 2009.

[23] G. Y. Wang, Z. Y. Hao, M. X. Hu, H. F. Zhang, and A. J. Chen, "Fingerprints of flos farfarae by high performance capillary electrophoresis," Chinese Journal of Experimental Traditional Medical Formulae, vol. 19, no. 13, pp. 97-100, 2013.

[24] D.-M. Liu, J. Chen, and Y.-P. Shi, "Screening of enzyme inhibitors from traditional Chinese medicine by magnetic immobilized $\alpha$-glucosidase coupled with capillary electrophoresis," Talanta, vol. 164, pp. 548-555, 2017.

[25] J. Chen, Q. Shi, Y. Wang, Z. Li, and S. Wang, "Dereplication of known nucleobase and nucleoside compounds in natural product extracts by capillary electrophoresis-high resolution mass spectrometry," Molecules, vol. 20, no. 4, pp. 5423-5437, 2015.

[26] J. Li, N. Ye, C. Gao, T. Zhou, and J. Ma, "Capillary coated with graphene oxide as stationary phase for the separation of brucine and strychnine by capillary electrophoresis," Journal of Chromatographic Science, vol. 53, no. 4, pp. 641-645, 2015.

[27] H. Zhao and Z. Chen, "Screening of neuraminidase inhibitors from traditional Chinese medicines by integrating capillary electrophoresis with immobilized enzyme microreactor," Journal of Chromatography A, vol. 1340, pp. 139-145, 2014.

[28] Q. Zhu, X. Xu, Y. Huang, L. Xu, and G. Chen, "Field enhancement sample stacking for analysis of organic acids in traditional Chinese medicine by capillary electrophoresis," Journal of Chromatography A, vol. 1246, pp. 35-39, 2012.

[29] X.-J. Chen, J. Zhao, Y.-T. Wang, L.-Q. Huang, and S.-P. Li, "CE and CEC analysis of phytochemicals in herbal medicines," Electrophoresis, vol. 33, no. 1, pp. 168-179, 2012.

[30] Z. Y. Hao, Y. J. Wu, W. Y. Zhao, G. Y. Wang, and A. J. Chen, "Analysis of three compouds in Flos Farfarae by Capillary Electrophoresis with Large-volume sample stacking," in Proceedings of the 8th National Micro Total Academic Conference on Analytical Systems, Third National Symposium on Microbiology and Biomass Separation, and 5th International Symposium on Microeconomics and Microsystems, 2013.

[31] E. Bermudo, O. Núñez, L. Puignou, and M. T. Galceran, "Analysis of acrylamide in food products by in-line preconcentration capillary zone electrophoresis," Journal of Chromatography A, vol. 1129, no. 1, pp. 129-134, 2006.

[32] L.-Y. Fan, T. He, Y.-Y. Tang et al., "Sensitive determination of barbiturates in biological matrix by capillary electrophoresis using online large-volume sample stacking," Journal of Forensic Sciences, vol. 57, no. 3, pp. 813-819, 2012.

[33] C. Tu, L. Zhu, C. H. Ang, and H. K. Lee, "Effect of $\mathrm{NaOH}$ on large-volume sample stacking of haloacetic acids in capillary zone electrophoresis with a low-pH buffer," Electrophoresis, vol. 24, no. 12-13, pp. 2188-2192, 2003.

[34] N. Woo, S.-K. Kim, and S. H. Kang, "Voltage-programmingbased capillary gel electrophoresis for the fast detection of angiotensin-converting enzyme insertion/deletion polymorphism with high sensitivity," Journal of Separation Science, vol. 39, no. 16, pp. 3230-3238, 2016.

[35] J. P. Quirino and A. M. Guidote, “Two-step stacking in capillary zone electrophoresis featuring sweeping and micelle to solvent stacking: II. Organic anions," Journal of Chromatography A, vol. 1218, no. 7, pp. 1004-1010, 2011.

[36] Y. H. Tak, G. W. Somsen, and G. J. De Jong, "Optimization of dynamic $\mathrm{pH}$ junction for the sensitive determination of amino acids in urine by capillary electrophoresis," Analytical and Bioanalytical Chemistry, vol. 401, no. 10, pp. 3275-3281, 2011.

[37] Z. Wang, C. Liu, and J. Kang, "A highly sensitive method for enantioseparation of fenoprofen and amino acid derivatives by capillary electrophoresis with on-line sample preconcentration," Journal of Chromatography A, vol. 1218, no. 13, pp. 17751779, 2011.

[38] D. Wu, M. Zhang, C. Zhang, and Z. Wang, "Flavonoids and phenolic acid derivatives from Flos Farfarae," Zhongguo Zhongyao Zazhi, vol. 35, no. 9, pp. 1142-1144, 2010.

[39] W. Y. Zhang, T. S. Yang, X. P. Wang, and W. X. Dang, "Determination of chlorogenic acid and rutin in coltsfoot flower by HPLC," Chinese Journal of Pharmaceutical Analysis, vol. 28, no. 12, pp. 2106-2108, 2008. 

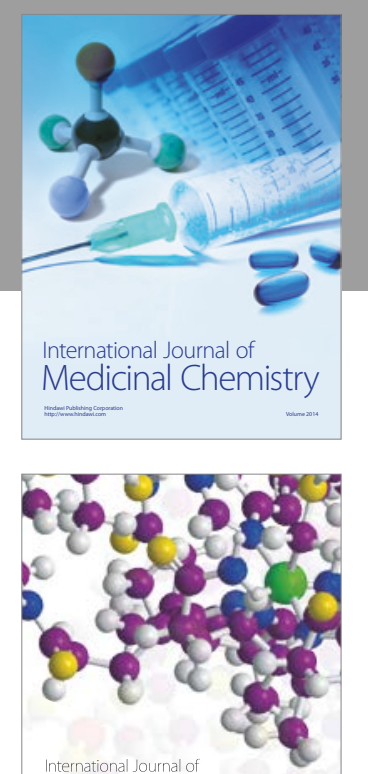

Carbohydrate Chemistry

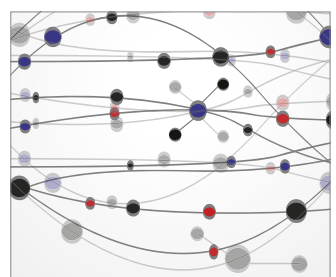

The Scientific World Journal
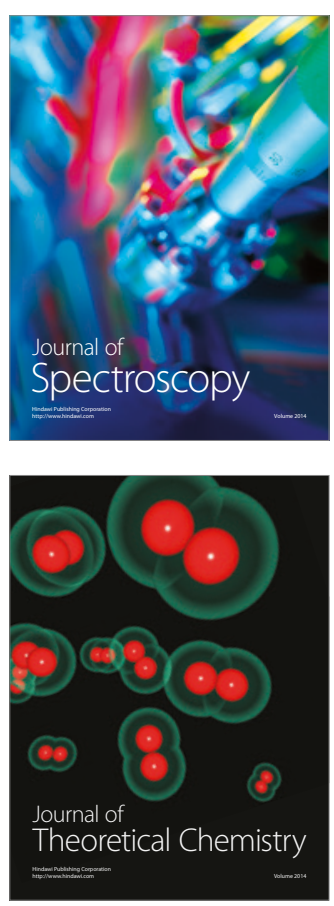
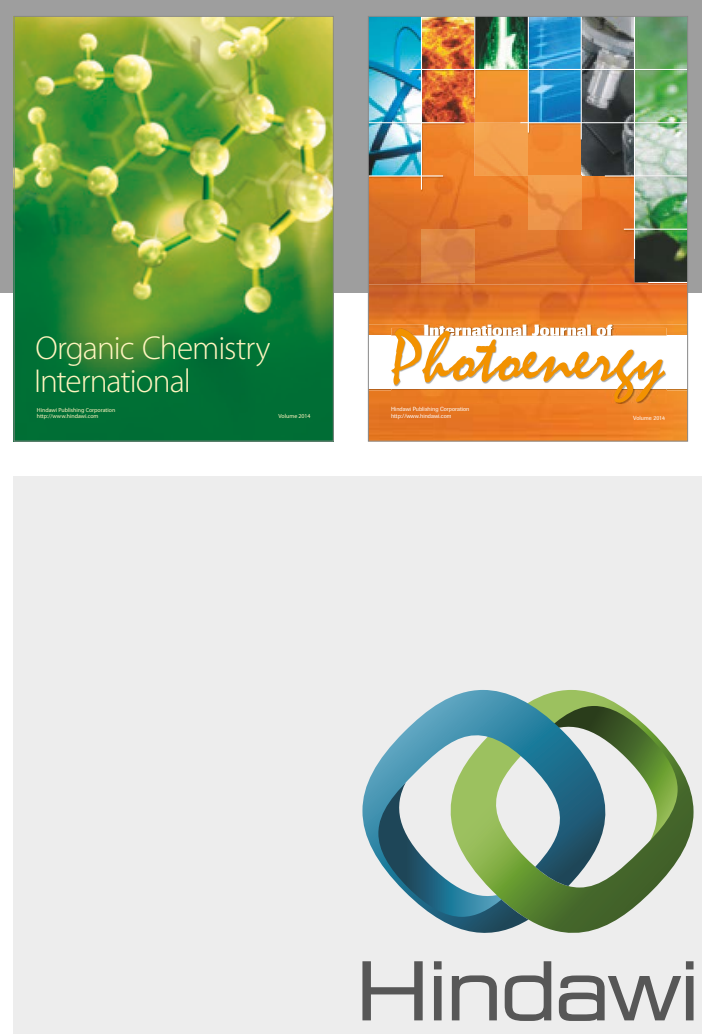

Submit your manuscripts at

https://www.hindawi.com

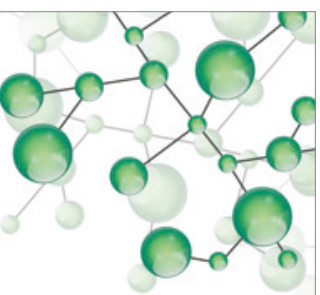

International Journal of

Inorganic Chemistry

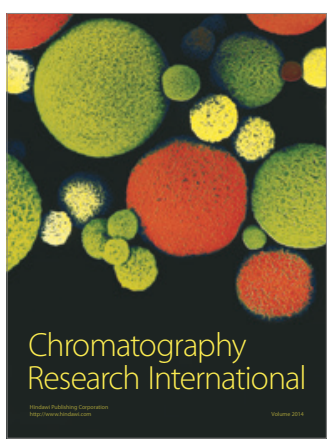

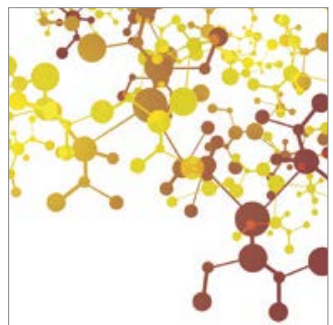

Applied Chemistry
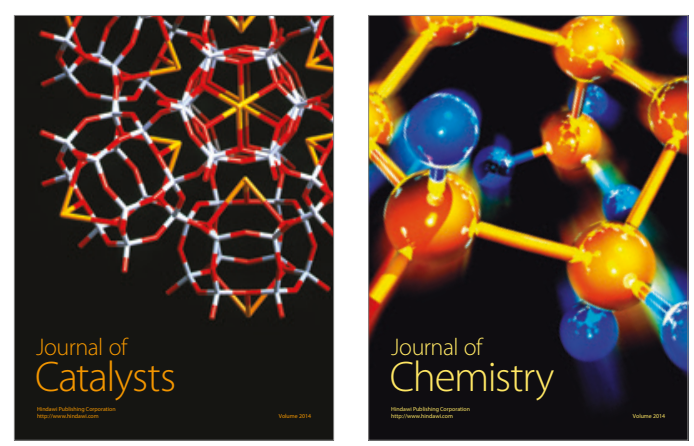
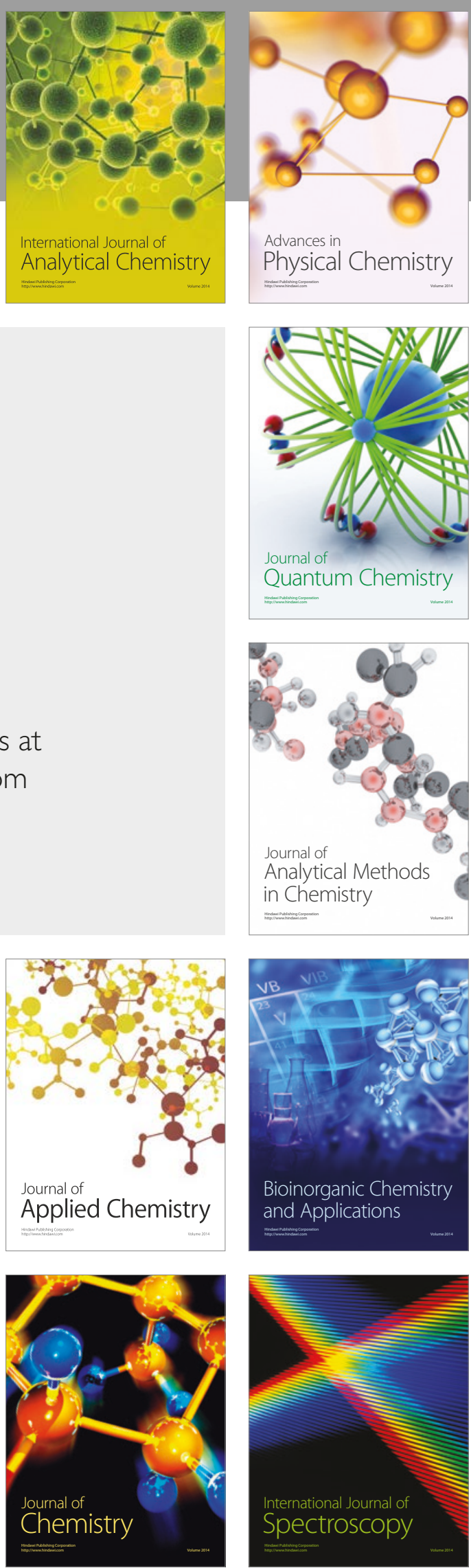